\title{
Implementation of a Double Continuous Flow Intersection in Riyadh
}

\author{
Kashif Bashir ${ }^{1}$, Rana Imam²*, Ammar Sharan ${ }^{3}$, Ala AlSoud ${ }^{4}$ \\ ${ }^{1}$ Kellogg Brown \& Root Pty Ltd., 3004 Melbourne, 441 Saint Kilda Road , Australia \\ 2 Department of Civil Engineering, The University of Jordan, 11942 Amman, Queen Rania Street, Jordan \\ ${ }^{3}$ Royal Commission for Riyadh City, 12512-3166 Riyadh, 7666 Amr Aldhamry Street, Saudi Arabia \\ ${ }^{4}$ Consolidated Consultants Group, 11372 Riyadh, 194 Mousa Bin Naseer St., Saudi Arabia \\ * Corresponding author, e-mail: r.imam@ju.edu.jo
}

Received: 21 May 2020, Accepted: 13 August 2020, Published online: 10 November 2021

\begin{abstract}
The continuous growth of population in the capital, coupled with increased auto ownership and dependence has worsened traffic conditions on Riyadh's road network. Conventional methods to address this increased demand could be costly and insufficient. There has been greater interest in using alternative measures to improve the performance and safety characteristics on main corridors, particularly those that arrive at signalized intersections. Heavy left turning traffic at these intersections is one of the main causes for delays. Previous research has investigated several types of alternative designs termed "unconventional" arterial intersection designs that could minimize the effect of left turning traffic. This paper provides decision makers with an objective assessment on the efficiency of implementing an unconventional intersection design, the Double Continuous Flow Intersection (DCFI) configuration, to improve the operational and safety characteristics of an existing major signalized arterial intersection in Saudi Arabia. In this study, the Kingdom Hospital Intersection in Riyadh was selected, as it is one of the most congested intersections in Riyadh. Using the collected traffic data, the micro-simulation program VISSIM was used to analyze and compare the efficiency of both configurations. When compared to the existing conventional signalized intersection design, it was found that the proposed Double Continuous Flow Intersection (DCFI) unconventional intersection design decreased the average delay per vehicle by 99 seconds. The proposed Double Continuous Flow Intersection configuration also improved the Level of Service at the intersection from level F (152 sec/veh average delay) to level D (53 sec/veh average delay).
\end{abstract}

\section{Keywords}

double continuous flow intersection, micro-simulation, unconventional intersection design, urban intersection, VISSIM software

\section{Introduction}

Traffic engineers and planners around the world are continuously challenged to meet mobility needs of a growing population with limited resources. At many urban junctions, congestion continues to worsen with both drivers and pedestrians experiencing long delays. For signalized intersections, it is noted practically at many intersections that high left turning traffic volumes significantly impact the performance of signals. Transportation engineers have implemented many conventional measures to ease this problem such as signal timing optimization, implementing double left turn lanes or widening intersections, and grade separation. However, such conventional measures fail under high traffic volumes and urge exploring unconventional intersection design ideas. These techniques help reduce congestion substantially by lowering the number of signal phases yet without eliminating any movements at the intersection.

Riyadh is one of the largest cities in the Arab world with a population exceeding 8.6 million inhabitants (General Authority of Statistics, KSA 2019). The city sprawled in a grid pattern with a large road network connected by ring roads, freeways, arterials, collectors and local roads. Being a populated city with high auto ownership, low fuel costs, and an absent modern public transport system encouraged the citizens to rely on private vehicles, hence putting a direct traffic burden on the road network. The problem became severe in the last decade when the freeways and interchanges in the metropolitan area were highly congested during the peak hours. Massive public transport projects are being developed to resolve the 
traffic issue such as the Metro system and the BRT, but they are both still in the construction and implementation stages. It will take almost a decade to change the commuting patterns in the city. In addition to such measurements, Riyadh Development Authority and Riyadh Municipality embarked upon a mission to improve the Level of Service of existing congested intersections; especially the major interchanges of the city center.

In this research, the Thumama Road and King Abdul Aziz Road intersection was chosen as a case study, to evaluate the operational performance of the unconventional DCFI design, being a major intersection in the commercial center of the city. The previous conventional design of the intersection had failed to handle the heavy volumes of traffic over the last couple of years especially during peak hours. Travel times increased extensively with users experiencing long queues of vehicles in multi-directions (up to $400 \mathrm{~m}$ ), and travel delays of around 152 seconds.

\section{Literature review}

Goldblatt et al. (1994) studied the Continuous Flow Intersections (CFI) in comparison with conventional intersection design. It was found that the CFI had a capacity of nearly $50 \%$ higher than conventional intersections. In addition, vehicle fuel consumption and emissions were reduced by more than $30 \%$, and at last, delay was found to be almost $20 \%$ of the conventional.

Dhatrak et al. (2010) analyzed the displaced left turn intersection (DLT) and the performance of the parallel flow intersection (PFI). The results indicated that on average the vehicles turning left experienced greater number of stops in a PFI than they would in the DLT design. Jagannathan and Bared (2004) studied the CFI, also known as the Crossover Displaced Left-turn (XDL) or Displaced Left Turn (DLT) intersection, and compared their operational performance to conventional intersection designs. Three cases were modeled:

- a four legged-intersection with four displaced left turns,

- a four legged-intersection with two opposing displaced left turns,

- and a T-intersection with one displaced left turn.

Two statistical models were built for the estimated average delay and average queue. The models were significant at $95 \%$ confidence level except for the delay model of the third case. Simulation results showed that the CFI design consistently outperforms a conventional intersection design, with the average intersection delay as a comparison parameter. Both models allow planners to compare the CFI with other types of intersections and the percentage of reduction in delays and queue length are computed for each case. The authors found that the CFI works at all levels of traffic and presents a suitable solution for intersections with high traffic volumes that aim to improve the LOS. El Esawey and Sayed (2011) evaluated and compared the performance of the XDL and the Upstream-Signalized Crossover intersection (USC) to a conventional design in Doha, Qatar. The results of this study indicated that the XDL and USC were consistent and that by increasing the secondary intersection to primary intersection distance will increase the capacity but delays will be a little higher at low volume conditions. Furthermore, the continuous flow intersection had approximately $90 \%$ higher capacity than the conventional intersection while that of the USC intersection is about $50 \%$ higher than the capacity of the conventional intersection.

The Federal Highway Administration's (FHWA) informal guide to signalized intersections (Robinson et al., 2000; Hughes et al., 2010) lists some disadvantages of the CFI, which include:

- Pedestrian acceptance (crossing only at main intersection - no midblock crossing);

- Driver acceptance (vehicles may be opposed by traffic on both sides);

- Snow removal issues;

- Breakdown of vehicles;

- Providing access to adjacent parcels.

Moreover, the footprint of a continuous flow intersection is greater than that of a conventional intersection because it requires right-turn lanes and acceleration lanes in each quadrant. In addition, according to Mier (Goldblatt et al., 1994), the construction cost of a CFI may be two to three times the cost of a standard intersection design due to increased right-of-way costs, and the need for additional, coordinated signal controllers. However, they are significantly cheaper than elevated design interchanges with savings ranging from 5:1 to 10:1 (Berkowitz et al., 1996). Naghawi et al. (2018) studied the implementation of the Superstreet unconventional intersection design at Tabarbour Intersection in Amman, Jordan. When compared to the existing conventional signalized intersection design, it was found that the proposed Superstreet unconventional intersection design decreased the average delay per vehicle by $70 \%$ and the maximum queue length by 
$72 \%$, so this resulted in the LOS improving from $\mathrm{F}$ to C. Likewise, Parsons (2007) has pointed out that the CFI is similar to the paraflow intersection in the left turn; which crosses over opposing lanes during the cross-street through movement phase. However, unlike the CFI, the paraflow junction leads to a smaller intersection with bypass turn lanes parallel to the lanes of the cross-street center. At a CFI, the left turns stop once, while left-turning vehicles at a paraflow stop multiple times, leading to more overall left-turn delay at a paraflow. The author compared four types of intersections, including the CFI, using VISSIM, RODEL and Synchro. The findings showed that both the CFI and the paraflow had a LOS "C" while the conventional intersections had a LOS "F".

Therefore, after conducting a thorough literature review on this topic, the authors found that so far there are no studies about a DCFI design anywhere in the world, so this will be the first example investigated and assessed.

\section{Site description}

Fig. 1 shows an aerial photo of the study area. The Kingdom intersection is located in the northern part of Riyadh, the capital of Saudi Arabia. The intersection is formed by Thumama Road and King Abdul Aziz Road. Both arterials are 6-lane divided major arterial roads carrying a total volume of around 8000 vehicles during peak hours from the city center. The Kingdom intersection is considered one of the most congested major intersections in the city. The Thumama Road has a $9 \mathrm{~km}$ signal-free section in the south-western direction of this intersection while on the other side, a signalized intersection falls at around $1.5 \mathrm{~km}$ from this junction. On the other hand, King Abdul Aziz Road has signalized intersections present on both sides, $2 \mathrm{~km}$ away of this junction. Fig. 1 depicts the study area.

The previous (conventional) configuration of the intersection was two back-to-back roundabouts joined by a 120 $\mathrm{m}$ road link, as shown in Fig. 2.

The main factors behind the high average delays (152 sec/veh) and poor LOS (Level F) of the intersection could be summarized briefly as:

- High traffic flows $8500 \mathrm{veh} / \mathrm{h}$ during the peak hour;

- Inadequate weaving lengths, where only a $120 \mathrm{~m}$ link separates the two roundabouts with 6 lanes, and drivers have to weave in through, left and right directions after crossing the northern side roundabout;

- Non-standardized geometry of both roundabouts with no properly defined entry and exit lanes. Major left-turn movements and through movements were prohibited;
- Skewed angle of intersection of about $70^{\circ}$ between Thumama Road and King Abdul Aziz Road resulting in smaller turning radii;

- The 3 lane U-turn being used to substitute the prohibited turning movement on the roundabouts;

- Driver confusion was inevitable because of the need to turn around both roundabouts in one-way direction with poor directional signage.

\section{Data collection}

Traffic counts were conducted at the roundabouts in order to establish the peak hours. In addition to traffic counts, a number of O-D (Origin-Destination) surveys were also carried out to get a clearer idea about the percentage of volumes going through or turning left from the joined roundabouts. Fig. 3 shows the approaches and the numbering system used to describe the traffic movements. Extensive visits to the site were also conducted during different times of the day and night to record personal observations and traffic trends. Automatic Traffic counts (ATC) were carried out on the main arterials for three consecutive working days over 24 hours to identify the traffic volume trends and identify the three peaks (AM, Noon, PM) needed to perform the manual turning movement counts, as shown in Fig. 3. The worst peak was the PM peak, so the manual counts were carried out accordingly. Table 1 shows the PM peak hour volumes for all intersection approaches, while Fig. 4 shows the fluctuation of volumes throughout the day. The O-D survey was needed to complement the turning movement manual counts and distinguish between movements due to the inadequate weaving lengths, where only a $120 \mathrm{~m}$ link separates the two roundabouts. Drivers are required to weave in through, right or left from the northern side of the roundabout; thus the movements numbered 5, 6, and 7 in Fig. 3 illustrate these southbound movements, respectively. In addition to the wide intersection right-of-way (ROW) and non-standardized geometry of both roundabouts major lefts and through movements were prohibited at the roundabout, so drivers had to travel an extra distance to reach their destinations while crossing the intersection.

The study area was divided into five Traffic Analysis Zones (TAZ) to carry out an O-D survey in order to understand the traffic movements and trends during the peak hour. Fig. 5 shows the TAZ in the study area of the intersection. Table 2 summarizes the O-D matrix developed for the study area. Zone 2 has internal trips due to the existing separated U-turn (fully protected) which generates 


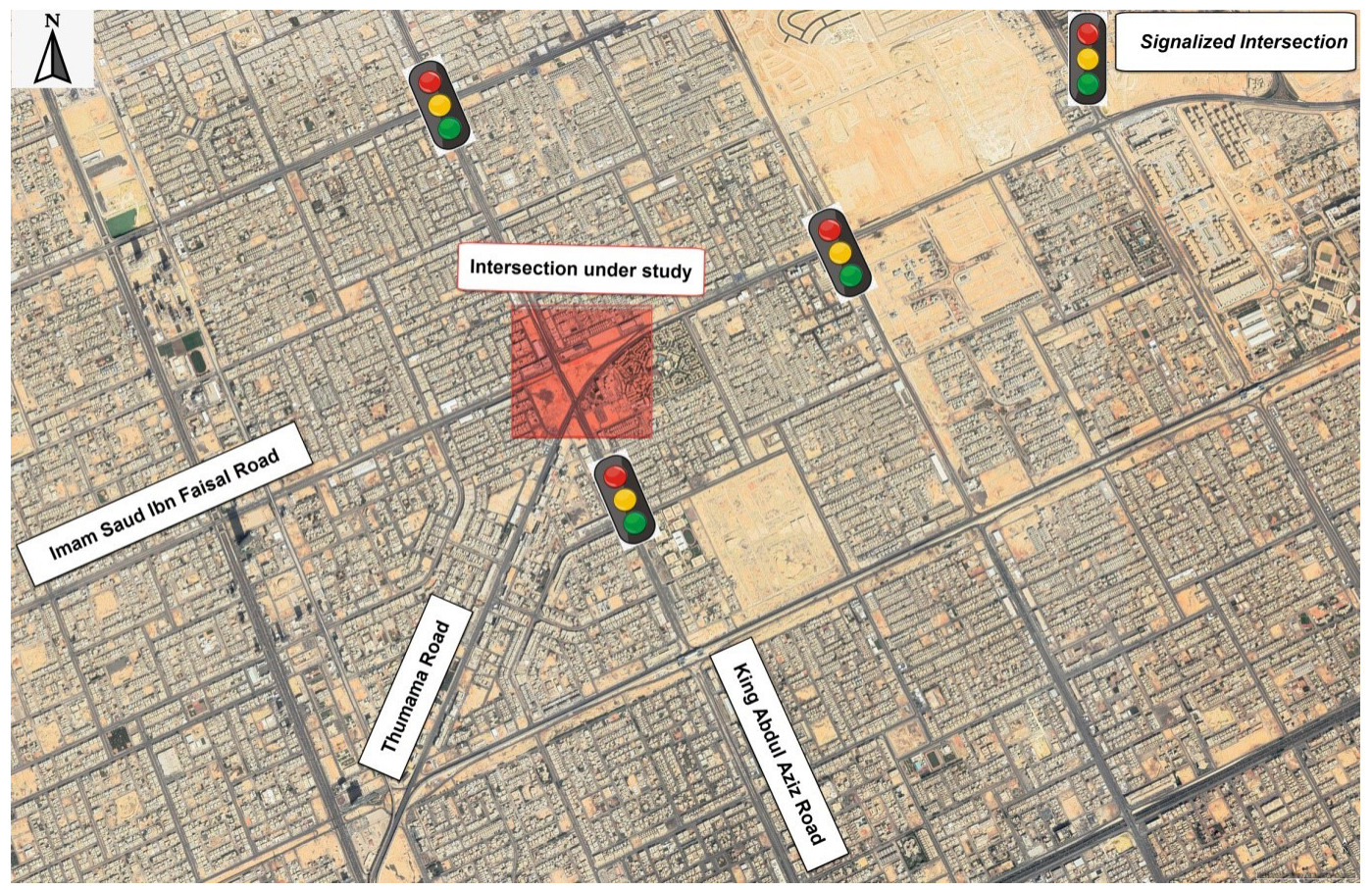

Fig. 1 Study area location in the city

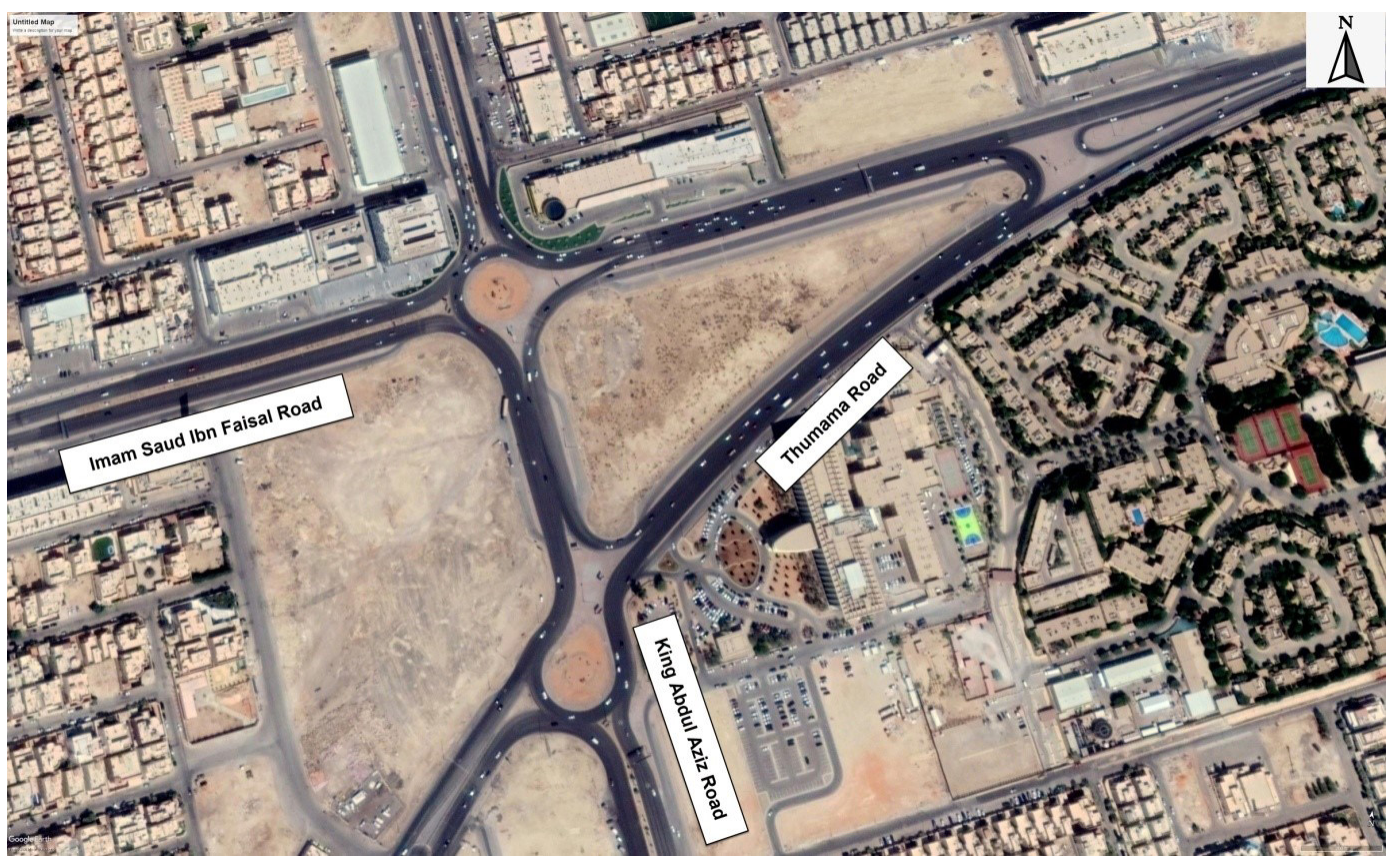

Fig. 2 Previous configuration of the intersection

internal trips, so any westbound vehicle using the separated U-turn only to change its direction is considered an internal trip within Zone2.

\section{Proposed solutions}

A number of conventional solutions were considered for this intersection but cons dominated the pros one way or another. Some of them are briefly described below.

\subsection{Grade separation}

The first conventional solution proposed was grade separation. One long flyover was proposed on King Abdul Aziz Road while 3 underpasses were proposed on Thumama Road. The rest of the movements were proposed to be operated at-grade through traffic signals. Despite the Grade Separation solution promising improvements, it was discarded due to: 


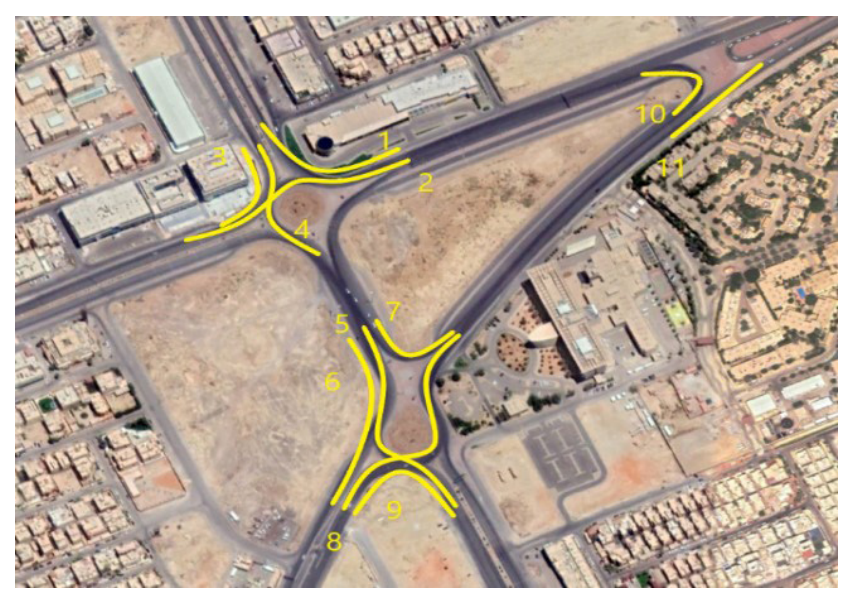

Fig. 3 Numbering of traffic movements

Table 1 Traffic volumes during the PM peak hour

\begin{tabular}{lccccc}
\hline & $\mathbf{1 6 : 3 0 - 1 6 : 4 5}$ & $\mathbf{1 6 : 4 5 - 1 7 : 0 0}$ & $\mathbf{1 7 : 0 0 - 1 7 : 1 5}$ & $\mathbf{1 7 : 1 5 - 1 7 : 3 0}$ & Total \\
\hline $\mathbf{1}$ & 552 & 489 & 557 & 578 & 2177 \\
$\mathbf{2}$ & 603 & 527 & 622 & 592 & 2346 \\
$\mathbf{3}$ & 12 & 11 & 14 & 10 & 50 \\
$\mathbf{4}$ & 450 & 447 & 470 & 435 & 1806 \\
$\mathbf{5}$ & 338 & 315 & 334 & 366 & 1358 \\
$\mathbf{6}$ & 370 & 386 & 362 & 405 & 1529 \\
$\mathbf{7}$ & 382 & 390 & 415 & 420 & 1614 \\
$\mathbf{8}$ & 416 & 437 & 459 & 456 & 1776 \\
$\mathbf{9}$ & 20 & 23 & 27 & 28 & 107 \\
$\mathbf{1 0}$ & 632 & 747 & 710 & 656 & 2755 \\
$\mathbf{1 1}$ & 865 & 982 & 856 & 796 & 3510 \\
\hline
\end{tabular}

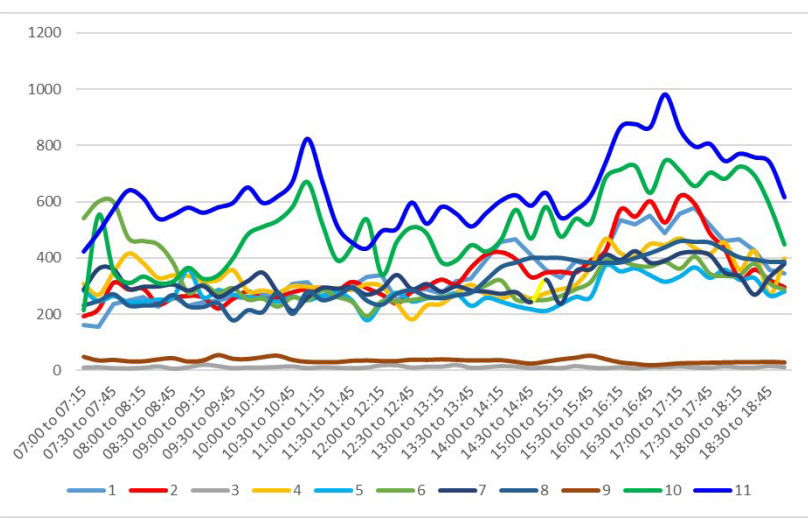

Fig. 4 Distribution of daily traffic volumes

- High implementation cost

- There are already 3 signalized junctions close to this intersection, which means that problem will simply be shifted to these intersections instead of being solved.

- The at-grade intersection design has accessibility issues.

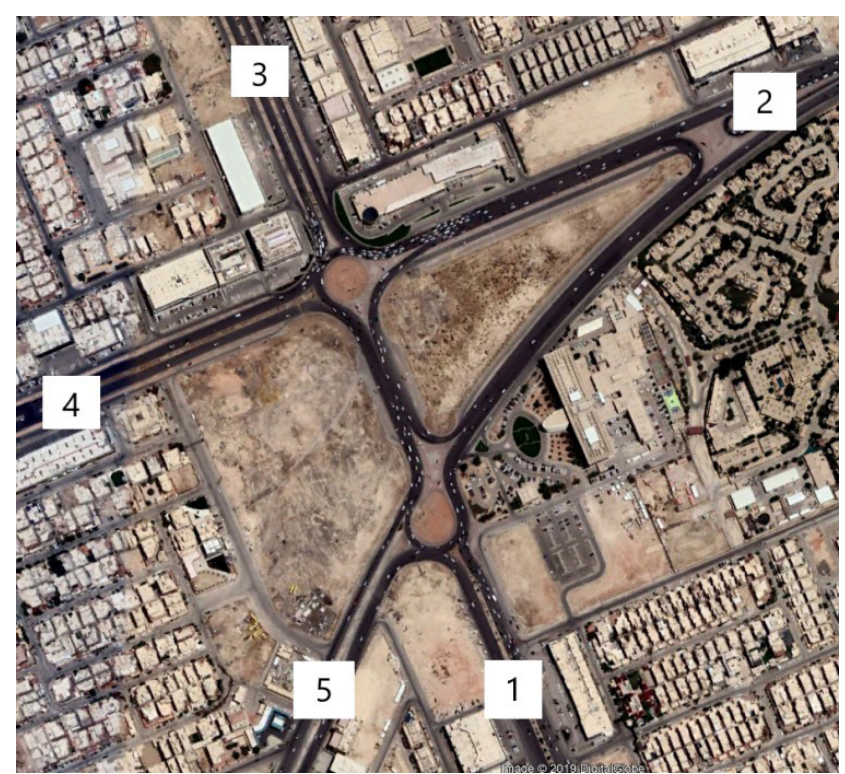

Fig. 5 Zoning system used for the O-D matrix survey

Table 2 O-D matrix survey results

\begin{tabular}{lcccccc}
\hline \multicolumn{7}{c}{ Table 2 O-D matrix survey results } \\
\hline $\mathbf{1}$ & $\mathbf{1}$ & $\mathbf{2}$ & $\mathbf{3}$ & $\mathbf{4}$ & $\mathbf{5}$ & Total \\
$\mathbf{2}$ & 0 & 704 & 753 & 189 & 112 & 1758 \\
$\mathbf{3}$ & 301 & 188 & 503 & 304 & 1083 & 2379 \\
$\mathbf{4}$ & 423 & 409 & 0 & 30 & 338 & 1200 \\
$\mathbf{5}$ & 462 & 415 & 375 & 0 & 277 & 1529 \\
Total & 1422 & 2502 & 2306 & 545 & 1810 & 8585 \\
\hline
\end{tabular}

\subsection{Signalized intersection}

Once the grade separation option was deemed unfit, the second conventional solution proposed was replacing the roundabouts with signalized solutions. A number of conventional solutions were proposed and tested at this location with 4-phase and 3-phase signal operations. However, none of these solutions were able to improve the LOS due to the extremely high volumes of traffic.

\subsection{Unconventional design proposal}

When all possible conventional intersection design options failed, unconventional design ideas were explored. Unconventional intersection designs (UID) aim at improving the operational performance, efficiency, and safety of intersections while reducing both the operation and construction costs. Several types of UID were explored including: CFI, Diverging Diamond Interchange (DDI), Median U-Turn, and Superstreet. As the traffic volumes are similarly distributed on all approaches, the Median U-Turn and the Superstreet options were eliminated since they both require that the through and left-turn movements of the minor roadway to be much less than those of 
the major roadway. Also, the intersection serves high leftturn volumes, so the Superstreet solution is not applicable since it does not permit through or direct left turn movements from the minor roadway. On the other hand, the DCFI serves arterials with high through and left-turn volumes and low demand for U-turn movements efficiently. Although the DDI could have produced good operational results, the through movements on the major road would need to be redirected with grade-separated geometric traffic solutions. This entails costly additions and would burden the design economically. Therefore, the DDI option was also dismissed.

The Continuous Flow Intersection (CFI) concept was adopted and tested. The CFI is an innovative idea in which the left turn movement is displaced before the main intersection across the opposite direction so that two concurrent left turns and two through movements could be operated on the same signal phase (Hummer and Reid, 2000). The concept could be applied on one crossing road (Single $\mathrm{CFI}$ ) or on both crossing roads (Double CFI) depending on the traffic conditions. The Double CFI operation is illustrated in Fig. 6 with the two phases depicted in green and red to differentiate between them.

Major benefits achieved by this configuration include:

- Reduction of number of signal phases from 4 to 2;

- Distribution of traffic all over the intersection, thus reducing congestion;

- Decreasing the number of conflict points; and

- Increasing signal green time substantially.

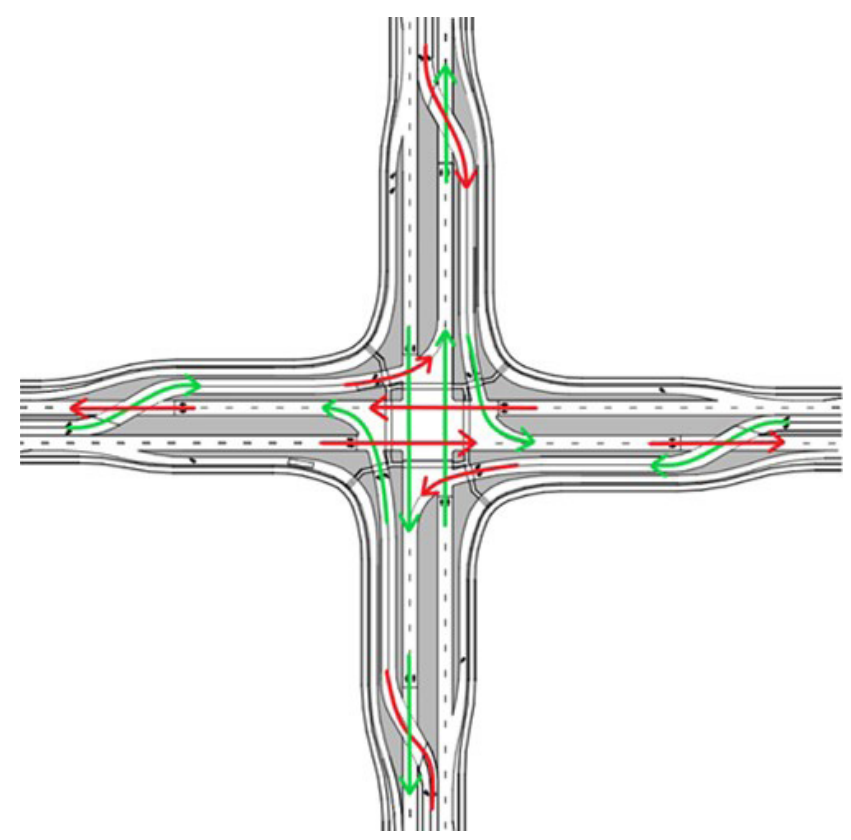

Fig. 6 The DCFI concept

\section{Model development}

Once the required data was available, a base case and a proposed CFI scenario were modeled, analyzed, and compared. In order to build the traffic simulation model for this intersection, the following steps were taken. To start with, the traffic volumes, compositions, and speed were defined. Also, speed reduction areas, and desired speed decisions points, and traffic control measures had to be specified. Later on, the traffic signals for proposed CFI with their phasing and timing plans were described.

\subsection{Base model}

From the collected traffic data and geometric data of the intersection, a base case scenario was built and analyzed using VISSIM 11. The parameters were adjusted to reflect the complex geometric conditions.

\subsection{Model validation and calibration}

The VISSIM simulation software was used to build the base model. However, the base model is only potentially as accurate as the calibration and validation processes undertaken during model development. Validation needs to be done using the most appropriate and efficient techniques, as accurately validated models form the base for proposed models. Toledo and Koutsopoulos (2004) proposed two methods of model validation; the visual method and the statistical method. The visual method of validation, where a graphical representation of the data from the real and simulated models are put side by side to see the difference between the real and simulated models, was not chosen as it is usually limited to an isolated road section or a traffic corridor. On the other hand, the statistical validation method was adopted in this research since it applies the goodness of fit measures, confidence intervals, and statistical tests to determine the similarity between the real and simulated models. The statistical method of validation was carried out in this research to validate the base model by using traffic volumes as a validation parameter. Other research also use the queue length as a validation parameter but it could not have been performed here due to the previous complex configuration of the intersection involving two connected roundabouts.

The traffic simulation was run for 10 times with different random seeds to take into account the randomness of vehicles arriving to the network. Average values for results were used for validation. Then the total number of vehicles arriving at destination were compared with the actual traffic counts. In addition to visually monitoring the 
simulation and comparing VISSIM simulation with actual condition on the site, statistical measures were used to see how much close the VISSIM model was to reality. One of the most used measures is the Root Mean-Square Percent Error RMSPE shown in Eq. (1) (Ni et al., 2004).

RMSPE $=\sqrt{\frac{\sum_{1}^{N}\left(\frac{Y_{\text {simulated }}-Y_{\text {observed }}}{Y_{\text {observed }}}\right)^{2}}{N}}$,

where:

- RMSPE: the root-mean-square percent error,

- $N$ : the number of simulation runs,

- $Y_{\text {simulated }}$ : the simulation run throughput volume,

- $Y_{\text {observed }}$ : the actual throughput volume.

RMSPE was found to be $10.12 \%$ which is less than the RMSPE threshold of $15 \%$ (Hourdakis et al., 2003), so the model is considered valid. Table 3 shows each simulation run with its random seed number and total number of vehicles modeled.

Another validation or goodness of fit measure adopted by researchers is the Thiel's inequality coefficient $(U)$, which is calculated using Eq. (2) and indicates the relative error. The $U$ value varies between 0 and 1 , where 0 indicates a perfect fit while 1 implies the worst possible fit. Based on the observed and simulated traffic volume data and number of simulation runs, the $U$ value was calculated using Eq. (2) (Hourdakis et al., 2003) and was found to be equal to 0.073 , this value is less than 0.3 which is the threshold $U$ value to attain a high accuracy for model building.

$$
U=\frac{\sqrt{\frac{1}{N} \sum_{n=1}^{N}\left(Y_{\text {simulated }}-Y_{\text {observed }}\right)^{2}}}{\sqrt{\frac{1}{N} \sum_{n=1}^{N}\left(Y_{\text {simulated }}\right)^{2}}+\sqrt{\frac{1}{N} \sum_{n=1}^{N}\left(Y_{\text {observed }}\right)^{2}}}
$$

Table 3 Simulation runs of the base case scenario

\begin{tabular}{lcc}
\hline SIMRUN & RANDSEED & Modelled volumes \\
\hline 1 & 45 & 9452 \\
2 & 46 & 7276 \\
3 & 47 & 9496 \\
4 & 48 & 9622 \\
5 & 49 & 9432 \\
6 & 50 & 9156 \\
7 & 51 & 9418 \\
8 & 52 & 9477 \\
9 & 53 & 9349 \\
10 & 54 & 8289 \\
\hline
\end{tabular}

Model calibration is the process where the model parameters are adjusted to closely represent the field situation. The travel time was used as it could be easily measured using a stopwatch, and the VISSIM software measures the travel time for all simulation/vehicles easily. The travel time during the rush hour was measured in the field ten times for ten different cars to get the average travel time in the field along King Abdul Aziz Road in the northbound direction. Travel times were measured and compared to the simulated average travel time for the same road segment at a percentage error of $4.8 \%$. This percentage error can be neglected.

\subsection{Proposed DCFI model development}

Once the base case and model validation were completed, the proposed DCFI solution was analyzed using the VISSIM software. The most challenging step during this analysis was fixing the displaced left crossovers. An adequate storage length needs to be chosen to avoid blocking the whole intersection. Long storage lengths (placing crossovers further) also affects the efficiency of the intersection. Therefore, a compromise value is required. Different distances were tested depending on the traffic volumes on each leg of the intersection and locations were finalized between $150 \mathrm{~m}$ to $200 \mathrm{~m}$ from the main intersection. There were 12 conflicting paths and 16 signals operating in each cycle. The speed at each leg varied due to geometry. Different sets of signal phasing were developed and checked in VISSIM to find the most optimized one.

The clearance time was highest at the main central intersection, with the All-Red time reaching 12 seconds. This is explained by the fact that the dedicated left turns on the Thumama Road were far from the main intersection due to the skew. Additional signals were proposed to stop the tail of each through phase on Thumama road. The All-Red time was reduced to 6 seconds after this addition. Figs. 7, 8, and 9 show the VISSIM simulation of the proposed design.

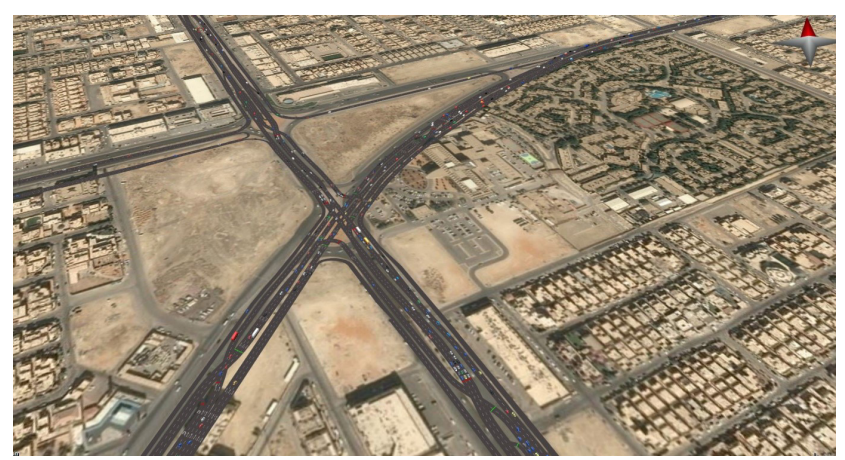

Fig. 7 General view of the DCFI 


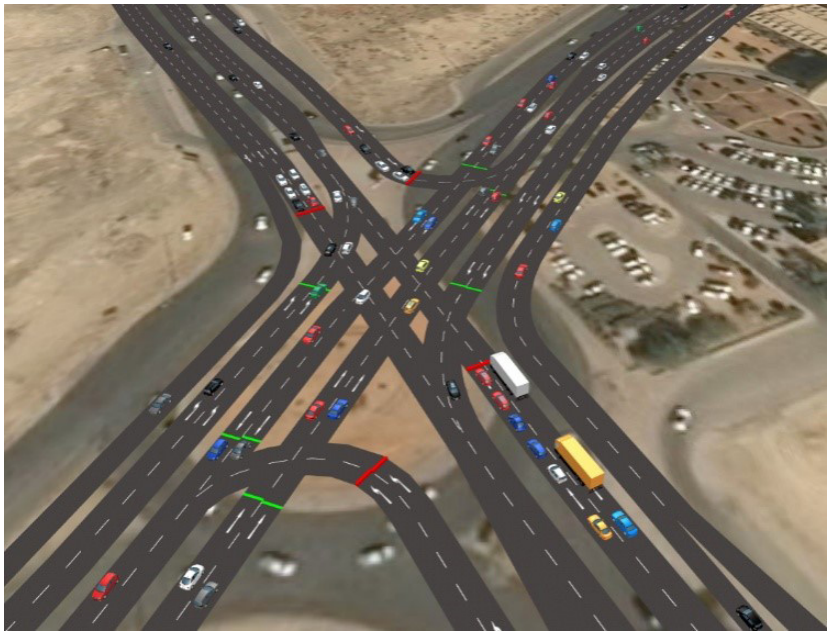

Fig. 8 Main intersection area

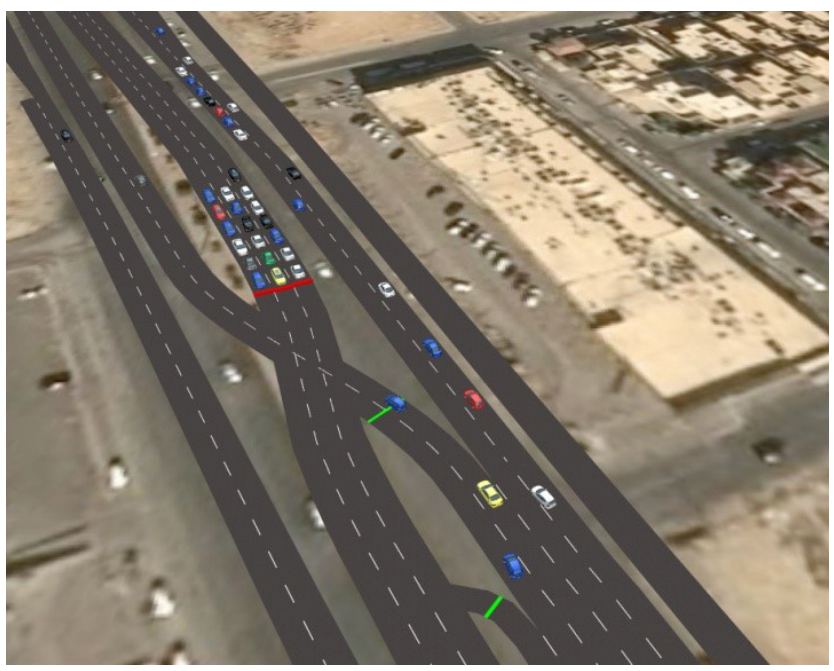

Fig. 9 Crossover intersection area

\section{Discussion of results}

To start with, Table 4 provides an aggregated comparison of both scenarios. It summarizes the VISSIM analysis results for both the existing conventional intersection and the proposed DCFI unconventional intersection.

Table 4 Traffic analysis results

\begin{tabular}{lcc}
\hline Scenario & Base case & DCFI \\
\hline Average delay (sec/veh) & 152.33 & 53.8 \\
Average number of stops & 8.62 & 1.48 \\
Maximum queue length $(\mathrm{m})$ & $\mathrm{N} / \mathrm{A}$ & 315.76 \\
Average speed $(\mathrm{km} / \mathrm{h})$ & 28.49 & 46.23 \\
Average stop delay $(\mathrm{sec})$ & 104.48 & 31.26 \\
Total distance traveled $(\mathrm{km})$ & 10,328 & 18,152 \\
Total travel time $(\mathrm{sec})$ & $1,784,013$ & $1,414,228$ \\
Total delay (sec) & $1,281,524$ & 479,390 \\
Total number of stops & 74,920 & 13,230 \\
Total stop delay (sec) & 858,720 & 278,528 \\
Vehicles served & 7,957 & 8,538 \\
\hline
\end{tabular}

The results include: the average delay per vehicle (in seconds/vehicle), average number of stops, maximum queue length (in $\mathrm{m}$ ), average speed (in $\mathrm{km} / \mathrm{h}$ ), average stop delay (in sec), total distance traveled (in $\mathrm{km}$ ), total travel time (in sec), total delay (in seconds), total numbers of stops, total stop delay (in seconds), and vehicles served (per hour). These performance measures reflect the traffic flow characteristics and discomfort levels experienced by drivers on interrupted-flow facilities. It was noted that the average speed increased from $28 \mathrm{~km} / \mathrm{h}$ to $46 \mathrm{~km} / \mathrm{h}$, which represents an improvement of more than $60 \%$. Although the total conflict points at the proposed DCFI are higher than those at the equivalent roundabout, these conflict points are dispersed over five two-phase signalized intersections. As a result, the total delay and the average number of stops are reduced by $65 \%$ and $83 \%$, respectively. Due to this improvement, the proposed DCFI enhances the capacity of the intersection by $7 \%$, as the number of served vehicles is increased to 8,538 vehicles compared to 7,957 vehicles at the existing conventional intersection.

Nevertheless, the intersection average delay has decreased significantly from (152 sec/veh) on the existing unconventional intersection design to $(53 \mathrm{sec} / \mathrm{veh})$ on the proposed DCFI unconventional intersection design. As a result, the intersection LOS has improved from Level $\mathrm{F}$ (forced or breakdown flow) to Level D (approaching unstable flow). The queue length was not considered a reliable measure of effectiveness or performance parameter due to non-standardized geometry of the two roundabouts, their close proximity, and the absence of traffic control devices in the base scenario, but it was measured for the implemented DCFI solution.

Fig. 10 provides the travel time comparison between the base case and the DCFI option for each individual direction. The highest percentage reduction was achieved for the South-East direction (72.2\%), followed by the

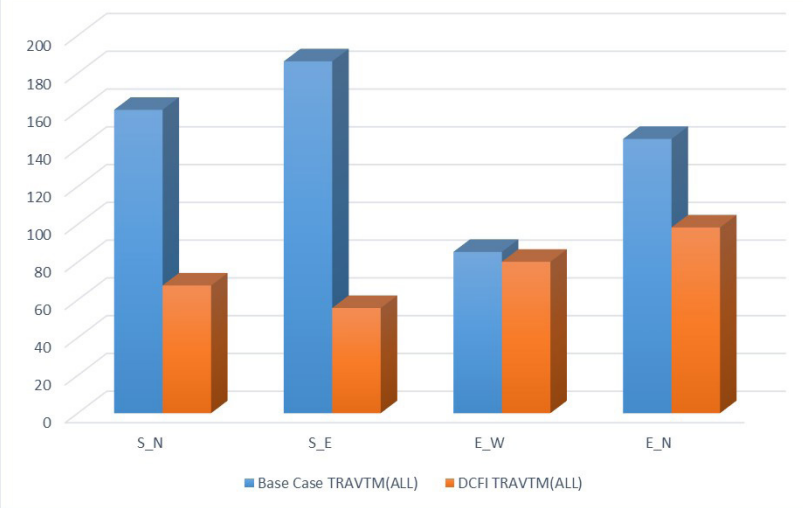

Fig. 10 Travel time comparison between the existing conditions and the proposed DCFI 
South-North (60.6 \%), East-North (34.2\%), and EastWest (3.75\%) movements.

Figs. 11 and 12 show the speed distribution for the prior conventional design and the proposed DCFI unconventional solution, respectively. The speed range for the conventional design was $(10-30) \mathrm{km} / \mathrm{h}$ but after the adoption of the DCFI design it ranged from $(50-80) \mathrm{km} / \mathrm{h}$.
Finally, queue lengths for the DCFI design scenario are shown in Fig. 13.

\section{Post implementation}

The first DCFI in the Middle East started operation on September 2018. It worked almost as simulated in VISSIM 11. On average, 140,000 vehicles used this

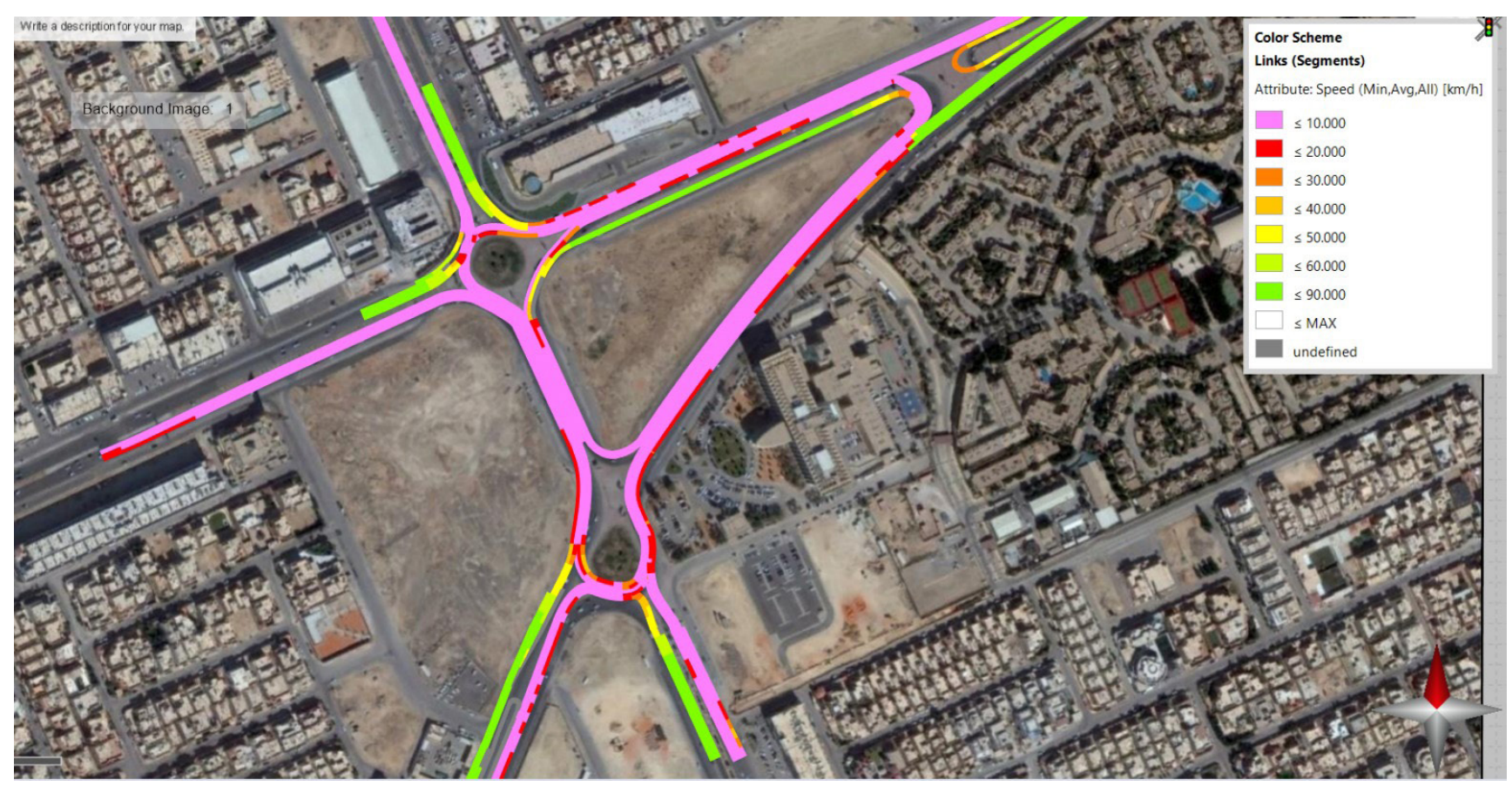

Fig. 11 Speed distribution map for the base case scenario

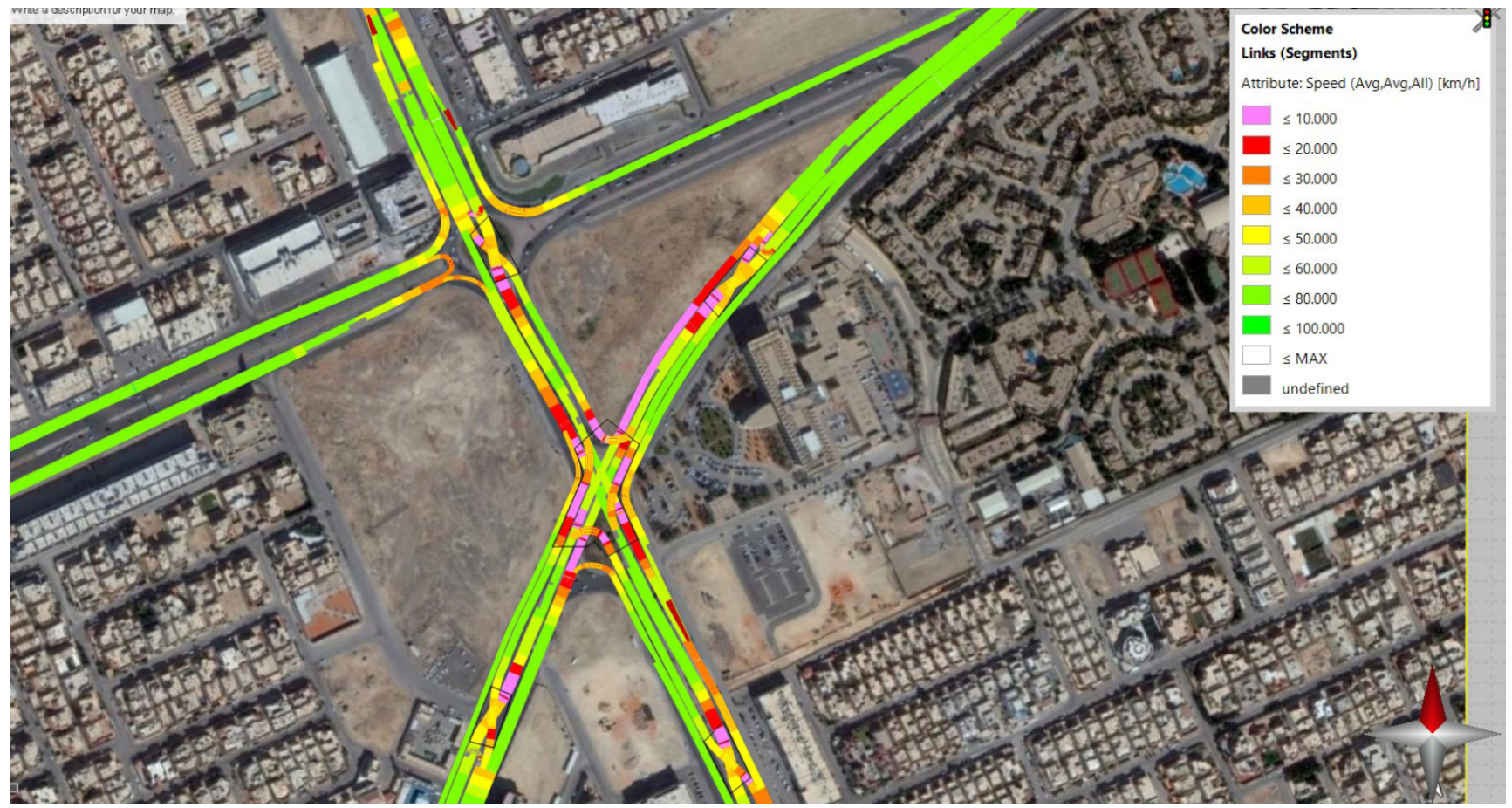

Fig. 12 Speed distribution map for the DCFI scenario 


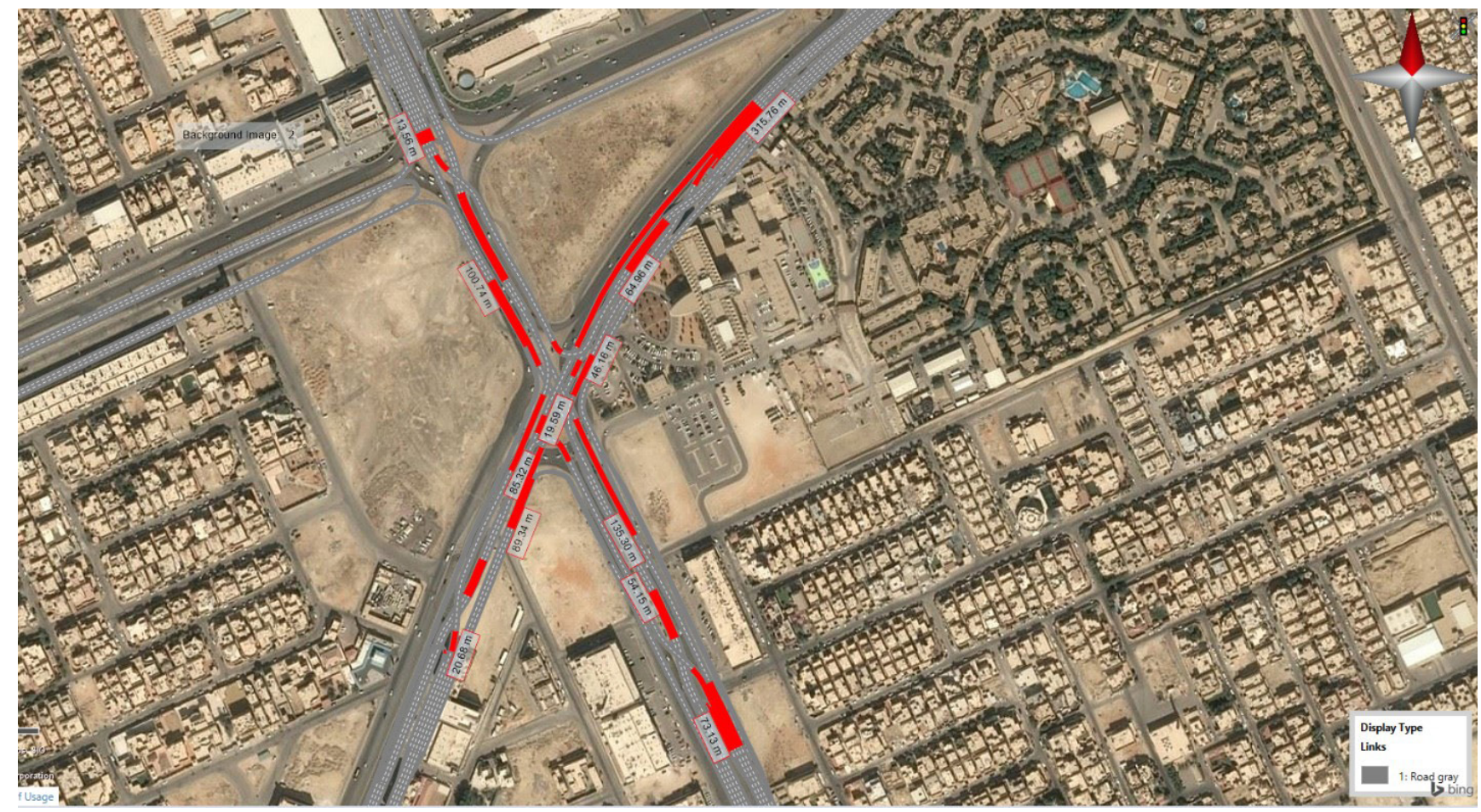

Fig. 13 DCFI queue lengths at the main and crossover intersections

intersection on a typical weekday. Fig. 14 shows the traffic volumes crossing this intersection on weekdays.

\section{Conclusions and recommendations}

This paper examined the adoption of the unconventional DCFI design on a major urban intersection in Riyadh. The main aim of this research is to objectively assess and compare this unconventional intersection's operational performance based on actual traffic data, and existing geometric conditions and intersection configurations. All of this above-mentioned exercise, from conceptualization of the idea till its successful implementation on site provided local consultants, relevant agencies and stakeholders, a unique opportunity of thinking out of the box. It also paved way for the implementation of unconventional concepts in other locations, due its capability of improving the level of service while saving costs. When compared to the existing conventional signalized intersection design, it was found that the proposed DCFI unconventional intersection design decreased the average delay per vehicle by 99 seconds. The

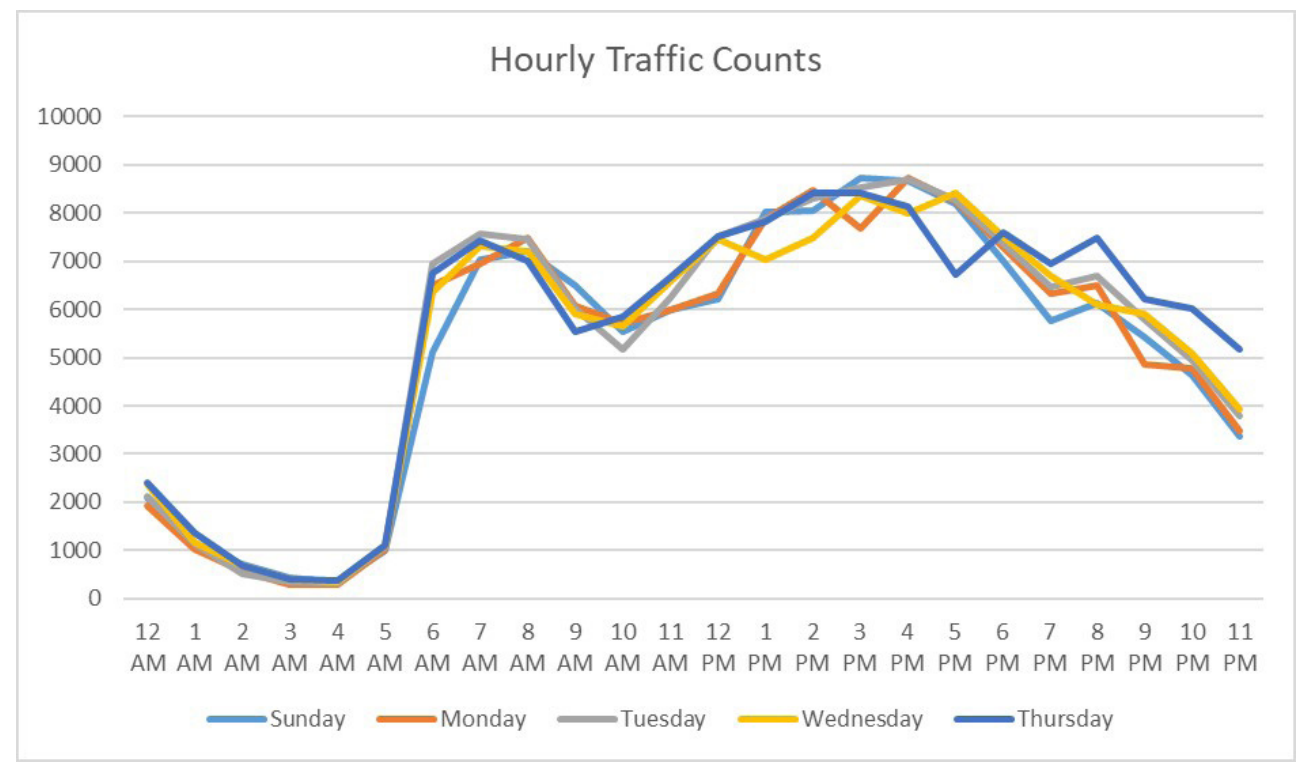

Fig. 14 Traffic volumes after the DCFI operation 
proposed configuration also improved the Level of Service at the intersection from level F (152 sec/veh average delay) to level D (53 sec/veh average delay). Travel times were compared between the base case and the DCFI option for each individual direction, where the highest percentage reduction was achieved for the South-East direction (72.2\%). It was noted that the average speed increased from $28 \mathrm{~km} / \mathrm{h}$ to $46 \mathrm{~km} / \mathrm{h}$, which represents an improvement of more than $60 \%$. Although the total conflict points at the proposed DCFI are higher than those at the equivalent roundabout, these conflict points are dispersed over five two-phase signalized intersections. As a result, the total delay and the average number of stops are reduced by $65 \%$ and $83 \%$, respectively. Due to this improvement, the proposed DCFI enhances the capacity of the intersection by $7 \%$, as the number of served vehicles is increased to 8,538 vehicles compared to 7,957 vehicles at the existing conventional intersection.

It is concluded that the proposed DCFI design offers benefits over the conventional design due to its efficient

\section{References}

Berkowitz, C., Bragdon, C., Mier, F. (1996) "Continuous flow intersection: A public private partnership", In: Vehicle Navigation and Information Systems Conference, 1996, Orlando, FL, USA, pp. 277-287. https://doi.org/10.1109/VNIS.1996.1623758

Dhatrak, A., Edara, P., Bared, J. G. (2010) "Performance Analysis of Parallel Flow Intersection and Displaced Left-turn Intersection Designs", Transportation Research Record: Transportation Research Board, 2171(1), pp. 33-43. https://doi.org/10.3141/2171-04

El Esawey, M., Sayed, T (2011) "Unconventional USC intersection corridors: evaluation of potential implementation in Doha, Qatar", Journal of Advanced Transportation, 45(1), pp. 38-53. https://doi.org/10.1002/atr.131

General Authority of Statistics "Statistical Yearbook 2019", General Authority of Statistics, Riyadh, Kingdom of Saudi Arabia.

Goldblatt, R., Mier, F., Friedman, J. (1994) "Continuous Flow Intersections", Institute of Transportation Engineers, ITE Journal, 64(7), pp. 35-42.

Hourdakis, J., Michalopoulos, P. G., Kottommannil, J. (2003) "A Practical Procedure for Calibrating Microscopic Traffic Simulation Models", Transportation Research Record: Journal of the Transportation Research Board, 1852(1), pp. 130-139. https://doi.org/10.3141/1852-17

Hughes, W., Jagannathan, R., Sengupta, D., Hummer, J. (2010) "Alternative Intersections/Interchanges: Informational Report (AIIR)", Federal Highway Administration, Turner-Fairbank Highway Research Center, McLean, VA, USA, Rep. FHWA-HRT-09-060.

Hummer, J. E., Reid, J. D. (2000) "Unconventional Left-Turn Alternatives for Urban and Suburban Arterial: An Update", The Urban Street Symposium, Dallas, TX, USA, Article number: E-3. two-phase operation. In view of the conclusions, the principal recommendations were as follows:

- Significant optimization in signal operations could be achieved if the signal phases are reduced from 4 to 2 , while maintaining all movements through the intersection.

- Substantial costs could be saved by at-grade intersection improvements by this concept, whereas the intersection level of service warranted grade separation.

- Highway authorities are encouraged to implement the same concept at other locations, if similar conditions are met.

As for limitations, there were no research studies or previous publications about DCFI. This limitation disallowed the authors to benchmark against other implementations to further develop and improve the model.

Jagannathan, R., Bared, J. G. (2004) "Design and Operational Performance of Crossover Displaced Left-Turn Intersections", Transportation Research Record: Journal of the Transportation Research Board, 1881(1), pp. 1-10. https://doi.org/10.3141/1881-01

Naghawi, H., AlSoud, A., AlHadidi, T. (2018) "The Possibility for Implementing the Superstreet Unconventional Intersection Design in Jordan", Periodica Polytechnica Transportation Engineering, 46(3), pp. 122-128.

https://doi.org/10.3311/PPtr.11635

Ni, D., Leonard II, J. D., Guin, A., Williams, B. M. (2004) "Systematic Approach for Validating Traffic Simulation Models", Transportation Research Record: Journal of the Transportation Research Board, 1876(1), pp. 20-31. https://doi.org/10.3141/1876-03

Parsons, G. F. (2007) "The Parallel Flow Intersection: A New Two-Phase Signal Alternative", Institute of Transportation Engineers, ITE Journal, 77(10), pp. 28-37.

Robinson, B. W., Rodegerdts, L., Scarborough, W., Kittelson, W., Troutbeck, R., Brilon, W., ... Jacquemart, G. (2000) "Roundabouts: An Informational Guide", U.S. Department of Transportation, Federal Highway Administration, McLean, VA, USA, Rep. FHWA-RD-00-067.

Toledo, T., Koutsopoulos, H. N. (2004) "Statistical Validation of Traffic Simulation Models", Transportation Research Record: Journal of the Transportation Research Board, 1876(1), pp. 142-150. https://doi.org/10.3141/1876-15 